\title{
Comparison of Clinical and Functional Outcomes Using Pectoralis Major and Cutaneous Free Flaps for Hypopharyngeal Squamous Cell Carcinoma
}

\author{
Taeyul Lee, Chulhoon Chung, Yongjoon Chang, Jaehyun Kim \\ Department of Plastic and Reconstructive Surgery, Hallym University College of Medicine, Seoul, Korea
}

Background The purpose of this study was to compare postoperative morbidities and functional outcomes of pectoralis major myocutaneous (PMMC) flap and cutaneous free flap reconstruction approaches in hypopharyngeal squamous cell carcinoma patients.

Methods We retrospectively reviewed records from 99 patients who underwent hypopharyngeal reconstruction with a cutaneous free flap $(n=85)$ or PMMC flap $(n=14)$ between 1995 and 2013. Morbidity was classified into hospitalization, medical, or flap-related complications. Functional outcomes were classified into oral re-alimentation and decannulation time.

Results The overall flap-related complication rate was higher in the PMMC flap group $(n=8$, $57.1 \% ; P=0.019)$, but the medical morbidity rate was higher in the cutaneous free flap group $(n=68,80 \% ; P=0.006)$. The rate of pneumonia was higher in the cutaneous free flap group $(n=48,56.5 \% ; P=0.020)$. Pulmonary premorbidity was the variable most significantly associated with pneumonia (odds ratio $=3.012, P=0.012$ ). There was no statistically significant difference in oral re-alimentation and decannulation time between the two groups.

Conclusions Although the functional superiority of free flaps has been reported in many studies, our results do not support this hypothesis. One limitation of our study is the relatively smaller flap size and fewer PMMC flap cases compared with the cutaneous free flap group. The low postoperative medical morbidity incidence rate in the PMMC flap group was clinically significant; however, the free flap group had more flap-related complications. Thus, PMMC flaps should be considered a viable option, especially for patients with pulmonary premorbidities.

Keywords Cutaneous free flap / Pectoralis major flap / Postoperative complication / Functional outcome / Hypopharynx

\author{
Correspondence: Chulhoon Chung \\ Department of Plastic and \\ Reconstructive Surgery, Hallym \\ University College of Medicine, 150 \\ Seongan-ro, Gangdong-gu, Seoul \\ 05355, Korea \\ Tel: +82-2-2224-2246 \\ Fax: +82-2-489-0010 \\ E-mail: c21ps@hanmail.net
}

No potential conflict of interest relevant to this article was reported.

Received: 30 Mar 2015 • Revised: 7 Jun $2015 \bullet$ Accepted: 23 Jun 2015

pISSN: 2234-6163 • elSSN: 2234-6171 • http://dx.doi.org/10.5999/aps.2015.42.5.608 • Arch Plast Surg 2015;42:608-613

\section{INTRODUCTION}

Hypopharynx reconstruction is among the most challenging head and neck reconstruction procedures due to its complexity and the possibilities of serious functional disabilities and poten- tial life-threatening complications. The goal of hypopharyngeal reconstruction is to preserve speech and swallowing functions without a permanent tracheostomy and to isolate vital structures from saliva and food. Hypopharyngeal squamous cell carcinoma (SCC) is a highly aggressive cancer associated with a 
poor prognosis since it is generally diagnosed at an advanced stage. The hypopharynx shares a wall with the larynx, and the latter is usually sacrificed during surgery to resect advanced hypopharyngeal SCC.

With the advent of microsurgery, a growing number of studies tout the superiority of free flaps [1-4]. Recently, in head and neck reconstruction, the prevalence of robot-assisted free flaps has increased [5]. Although the cutaneous free flap tends to be preferred over the pectoralis major myocutaneous (PMMC) flap in head and neck cancer, the use of the PMMC flap persists as a common reconstructive procedure in many centers [2,6-9]. As most centers usually perform one type of flap surgery, few studies have directly compared systemic morbidities including flap survival and functional outcomes between reconstructive types. Consequently, the choice of flap seems to be dependent on surgeons' preference and experience.

First reported by Ariyan [10] as an island pedicle flap in 1979, the pectoralis major flap has been the standard for head and neck reconstruction because it is stable and can be promptly and easily elevated [11]. The radial forearm free flap (RFFF) was first described by Yang et al. [11] in 1981. This approach offers a large amount of thin, pliable skin that conforms well to the native contours of the recipient site. The long pedicle allows anastomoses to be performed in either the ipsilateral or contralateral neck. The anterolateral thigh (ALT) free flap has recently gained popularity for head and neck reconstruction because it provides a larger surface area of expendable tissue with a long vascular pedicle. In addition, the distance from the head and neck allows this flap to be harvested at the same time as the ablative procedure is carried out. The purpose of this study is to compare two types of reconstruction approaches, PMMC flap and cutaneous free flap (RFFF and ALT free flap), for treating hypopharyngeal cancer in terms of systemic morbidity and functional outcome.

\section{METHODS}

We retrospectively reviewed a total of 113 patients with hypopharyngeal SCC who underwent surgery as a primary treatment and immediate reconstruction with cutaneous free flaps or pectoralis major flaps at the departments of plastic and reconstructive surgery and otolaryngology head and neck surgery of our medical institution between 1995 and 2013. All of the reviewed reconstructive operations were performed by a single experienced reconstructive team to minimize the effect of the learning curve and measurement bias. We limited the patient population to hypopharyngeal SCC, excluding other types of carcinoma to reduce the effect of surgical variables. Salvage cases and patients lacking at least 6 months of functional outcome data after sur- gery (14 cases) were excluded. Consequently, 99 hypopharyngeal SCC patients were retrospectively analyzed. The study group comprised 98 males and 1 female (mean age, 62.5 years; range, 36 to 86 years) including 46 patients (46.5\%) who underwent laryngeal preservation as a primary treatment. This approach was contraindicated in patients with pyriform apex invasion or lesions extending to the postcricoid area.

Two types of reconstruction approaches were compared with regard to systemic morbidity and functional outcome. Systemic morbidities were classified as flap-related complications, postoperative systemic medical morbidities, and length of hospitalization while functional outcomes were classified as oral re-alimentation and decannulation time for swallowing and speech functions. Flap-related complications were classified into major complications requiring an operation and minor complications addressed with conservative treatment. Flap-related complications were further divided into recipient site and donor site morbidities. If more than one complication was involved, the more serious or ultimate complication was included in the analysis. Hospitalization duration was measured in days and defined as the length of time that elapsed between surgery and discharge. If there was no discharge between the operation and other cancer treatments, the first date of postoperative chemo- or radiotherapy was defined as the discharge date.

For effective comparison, functional outcomes were classified into laryngeal preservation and total/near-total laryngectomy groups. Gastrostomy tube dependence is another reliable indication of swallowing function and was defined as the need for a percutaneous endoscopic gastrostomy tube for nutrition. The decannulation time was measured in days; short decannulation time is a useful indicator for faster recovery of swallowing and speech functions. In our study, complication risk factors included sex, smoking history, operative duration, age $\geq 65$ years old and major comorbid conditions (diabetes mellitus, hypertension, cerebrovascular accident, pulmonary and cardiovascular diseases), and we confirmed their correlations with systemic complications. The total operative duration was measured in hours and defined as the length of time that elapsed between intubation and extubation.

Statistical analyses were performed using PASW ver. 18.0 (SPSS Inc., Chicago, IL, USA). P-values for each variable were calculated using Fisher exact test, the independent t-test, non-parametric Mann-Whitney test, or logistic regression analysis. For the reconstruction-type comparison, Fisher exact test was used for comparing proportions. The non-parametric Mann-Whitney test and independent $\mathrm{t}$-test were used to compare medians and means. Logistic regression analysis was performed to explore the prognostic roles of relevant variables and estimate the odds 
ratio (OR) for postoperative medical morbidity. The normality distribution for continuous variables was validated using the Shapiro-Wilk test. Differences were considered statistically significant at $\mathrm{P}<0.05$.

\section{RESULTS}

This study was designed to balance demographic and clinical variables and treatment. There were no significant differences in the incidences of comorbid conditions or any other variables between the two groups. However, PMMC flaps were more frequently used to shorten the operative duration due to a poor general condition caused by severe diabetes, hypertension, ischemic heart disease, or liver cirrhosis (Table 1).

The cutaneous free flap group comprised 85 patients who underwent RFFF ( 75 cases) or ALT free flap (10 cases), and the PMMC flap group consisted of 14 patients. Hypopharyngeal SCC was classified as stage I, II, III, and IV in 0 patients $(0.0 \%)$, 6 patients (6.1\%), 12 patients (12.1\%), and 81 patients $(81.8 \%)$, respectively. That the vast majority of patients were stage IV demonstrates that our cohort was made up of advanced cases that required major reconstruction. A total of 46 patients (46.5\%) underwent laryngeal preservation as the primary treatment (RFFF: $\mathrm{n}=42,56.0 \%$; ALT free flap: $\mathrm{n}=2,20.0 \%$; and PMMC: $\mathrm{n}=2$, $14.3 \%)$.

In all cases, the flap survived without any vascular crisis complications. The size of the skin paddle ranged from 20 to 168 $\mathrm{cm}^{2}$, and the mean flap size was $80.4 \mathrm{~cm}^{2}$. As shown in Table 1 , the mean size was significantly larger in the cutaneous free flap group $\left(83.7 \mathrm{~cm}^{2}\right)$ than in the PMMC flap group $\left(58.7 \mathrm{~cm}^{2}, \mathrm{P}=\right.$ 0.001 ). The skin paddles were the largest in cases where the cir-

\begin{tabular}{|c|c|c|}
\hline Characteristic & $\begin{array}{l}\text { Cutaneous free flap } \\
\qquad(\mathrm{n}=85)\end{array}$ & $\begin{array}{l}\text { Pectoralis major flap } \\
\qquad(\mathrm{n}=14)\end{array}$ \\
\hline Male & $84(98.8)$ & $14(100.0)$ \\
\hline Female & $1(1.2)$ & 0 \\
\hline Mean age (yr) & $62.4(36-77)$ & $62.9(44-86)$ \\
\hline Mean size of flap $\left(\mathrm{cm}^{2}\right)^{\mathrm{b})}$ & 83.7 (25-168) & $58.7(20-96)$ \\
\hline Major comorbidities & $54(63.5)$ & $10(71.4)$ \\
\hline Smoking history & $76(89.4)$ & $12(85.4)$ \\
\hline \multicolumn{3}{|l|}{ Staging of cancer } \\
\hline Stage I & 0 & 0 \\
\hline Stage II & $6(7.1)$ & $4(28.6)$ \\
\hline stage III & $10(11.8)$ & $2(14.3)$ \\
\hline Stage IV & 69 (81.2) & $12(85.7)$ \\
\hline \multicolumn{3}{|c|}{$\begin{array}{l}\text { Values are presented as number (\%). } \\
\text { a)Both recurrent and secondary primary squamous cell carcinoma were excluded; } \\
\text { bjValues presented as mean (range), the normality variation for each group was } \\
\text { validated using the Shapiro-Wilk test. } P=0.001 \text { compared between the two flap } \\
\text { groups, as determined by an independent t-test. }\end{array}$} \\
\hline
\end{tabular}

cumferential defect of the hypopharynx was reconstructed with a tubed ALT free flap.

The flap-related complications included 27 problems (27.3\%) at recipient sites such as fistula formation $(\mathrm{n}=11,11.1 \%)$, infection $(\mathrm{n}=7,7.1 \%)$, dehiscence $(\mathrm{n}=5,5.1 \%)$, partial flap necrosis $(n=2,2.0 \%)$, stenosis $(n=1,1.0 \%)$, and seroma $(n=1,1.0 \%)$. Among them, 14 and 13 cases involved major and minor complications, respectively. The overall incidences of flap-related complications and dehiscence were significantly higher in the PMMC flap group than in the cutaneous free flap group. Although there were seemingly greater proportions of patients in the cutaneous free flap group and PMMC flap group with fistula formation and major complications, respectively, neither difference was statistically significant. The incidence of flap-related complications was tested using Fisher exact test (Table 2).

The flap-related complications included 16 donor site issues (16.2\%), such as skin graft partial necrosis $(n=14,14.1 \%)$ and dehiscence $(n=2,2.0 \%)$. Of those patients, major and minor complications occurred in 12 and 2 cases, respectively. Despite the trend towards greater skin graft partial necrosis and dehiscence in the cutaneous free flap group and PMMC flap group, respectively, neither difference was statistically significant (Table 2).

The overall postoperative medical morbidity incidence was significantly higher in the cutaneous free flap group than in the PMMC flap group ( $80.0 \%$ vs. $42.9 \%$, respectively, $\mathrm{P}=0.006)$. The incidence of postoperative pulmonary problems was significantly higher in the cutaneous free flap group compared to the PMMC flap group (63.5\% vs. $28.6 \%$, respectively, $\mathrm{P}=0.019$ ). Specifically, a much larger percentage of the cutaneous free flap group developed pneumonia compared to the PMMC flap group ( $56.5 \%$ vs. $21.4 \%$, respectively, $\mathrm{P}=0.020$ ). The incidence rates

\begin{tabular}{|c|c|c|c|}
\hline Complication & $\begin{array}{l}\text { Cutaneous } \\
\text { free flap } \\
(n=85)\end{array}$ & $\begin{array}{l}\text { Pectoralis } \\
\text { major flap } \\
(n=14)\end{array}$ & P-value ${ }^{a)}$ \\
\hline \multicolumn{4}{|l|}{ Recipient site } \\
\hline Overall complication & $19(22.4)$ & $8(57.1)$ & 0.019 \\
\hline Stenosis & $1(1.2)$ & 0 & 0.859 \\
\hline Partial necrosis & $1(1.2)$ & $1(7.1)$ & 0.264 \\
\hline Fistula & $9(10.6)$ & $2(14.3)$ & 0.652 \\
\hline Dehiscence & $1(1.2)$ & $4(28.6)$ & 0.001 \\
\hline Infection & 7 (8.2) & 0 & 0.589 \\
\hline Seroma & 0 & $1(7.1)$ & 0.141 \\
\hline Major complication & $11(12.9)$ & $3(21.4)$ & 0.413 \\
\hline \multicolumn{4}{|l|}{ Donor site } \\
\hline Overall complication & $14(16.5)$ & $2(14.3)$ & 1.000 \\
\hline Partial necrosis (STSG $\left.{ }^{b}\right)$ & $13(15.3)$ & $1(7.1)$ & 0.685 \\
\hline Dehiscence & $1(1.2)$ & $1(7.1)$ & 0.264 \\
\hline Major complication & $11(12.9)$ & $1(7.1)$ & 1.000 \\
\hline
\end{tabular}


Table 3. Postoperative medical morbidity in patients

\begin{tabular}{|lccc|}
\hline $\begin{array}{l}\text { Postoperative } \\
\text { medical morbidity }\end{array}$ & $\begin{array}{c}\text { Cutaneous } \\
\text { free flap } \\
(\mathbf{n = 8 5})\end{array}$ & $\begin{array}{c}\text { Pectoralis } \\
\text { major flap } \\
(\mathbf{n = 1 4 )}\end{array}$ & P-value $^{\text {a) }}$ \\
\hline $\begin{array}{l}\text { Postoperative medical } \\
\text { complications }\end{array}$ & $68(80.0)$ & $6(42.9)$ & 0.006 \\
Pulmonary complication & $54(63.5)$ & $4(28.6)$ & 0.019 \\
$\quad$ Pneumonia & $48(56.5)$ & $3(21.4)$ & 0.020 \\
$\quad$ Atelectasis & $6(7.0)$ & $1(7.1)$ & 0.465 \\
Gastrointestinal problem & $1(1.2)$ & $1(7.1)$ & 0.264 \\
Delirium & $16(18.8)$ & $1(7.1)$ & 0.453 \\
Cardiovascular problem & $2(2.4)$ & 0 & 0.736 \\
\hline $\begin{array}{l}\text { Values are presented as number (\%). } \\
\text { a)Fisher exact test. }\end{array}$ & & \\
\hline
\end{tabular}

\section{Table 4. Risk factors in patients}

\begin{tabular}{|lccc|}
\hline Risk factor & $\begin{array}{c}\text { Cutaneous } \\
\text { free flap } \\
(\mathbf{n}=\mathbf{8 5})\end{array}$ & $\begin{array}{c}\text { Pectoralis } \\
\text { major flap } \\
(\mathbf{n}=14)\end{array}$ & P-value ${ }^{\text {a) }}$ \\
\hline Age $\geq 65$ yr & $39(45.9)$ & $7(50.0)$ & $0.782^{\text {a) }}$ \\
Mean of operation time $(\mathrm{hr})^{\mathrm{b})}$ & $15.8 \pm 1.7$ & $12.7 \pm 1.9$ & $0.001^{\mathrm{c}}$ \\
Smoking history & $77(90.6)$ & $11(78.6)$ & $0.186^{\mathrm{a})}$ \\
Alcohol history & $76(89.4)$ & $12(85.7)$ & $0.652^{\mathrm{a}}$ \\
Pulmonary premorbidities & $28(32.9)$ & $3(21.4)$ & $0.539^{\mathrm{a})}$ \\
Diabetes mellitus & $13(15.3)$ & $2(14.3)$ & $1.000^{\mathrm{a})}$ \\
Hypertension & $28(32.9)$ & $3(21.4)$ & $0.539^{\mathrm{a})}$ \\
Liver cirrhosis & $14(16.5)$ & $3(21.4)$ & $0.703^{\mathrm{a}}$ \\
\hline
\end{tabular}

Values are presented as number (\%).

a)Fisher exact test; ")Described as mean \pm standard deviation. The normality variation for each group was validated using the Shapiro-Wilk test; "IIndependent t-test.

of postoperative delirium, atelectasis, and cardiovascular complications were higher in the cutaneous free flap group, but the differences were not statistically significant. Differences in postoperative medical morbidity rates were compared with Fisher exact test (Table 3).

We next assessed the relationships between the two reconstructive approaches and risk factors affecting postoperative morbidity (i.e., smoking history, alcohol history, age $\geq 65$ years old, operative duration, and premorbidities). Only the mean operative duration was significantly longer in the cutaneous free flap group compared to the PMMC flap group (15.8 vs. 12.7 hours, respectively, $\mathrm{P}=0.001)$. Interestingly, most risk factors showed no correlation with either reconstruction approach. The risk factors were tested using Fisher exact test and the independent $t$-test (Table 4).

The risk factors that were significantly associated with postoperative pneumonia were pulmonary premorbidity $(\mathrm{OR}=3.012)$, smoking history $(\mathrm{OR}=2.682)$, operative duration $(\mathrm{OR}=2.327)$, and older age $(\mathrm{OR}=2.145)$. All independent valuables were statistically significant predictors of postoperative pneumonia $(\mathrm{P}<$
Table 5. Logistic regression analysis of the probability of postoperative pulmonary complications

\begin{tabular}{|c|c|c|c|c|}
\hline \multirow{2}{*}{$\begin{array}{l}\text { Independent } \\
\text { valuable }\end{array}$} & \multicolumn{2}{|l|}{ Pneumonia } & \multicolumn{2}{|l|}{ Atelectasis } \\
\hline & $\begin{array}{l}\text { Odds ratio } \\
(95 \% \mathrm{Cl})\end{array}$ & $\begin{array}{c}\mathrm{P}- \\
\text { value }\end{array}$ & $\begin{array}{l}\text { Odds ratio } \\
(95 \% \mathrm{Cl})\end{array}$ & $\begin{array}{c}\mathrm{P}- \\
\text { value }\end{array}$ \\
\hline Surgery timea) & 2.327 (1.083-5.634) & 0.031 & $0.951(0.634-1.416)$ & 0.630 \\
\hline Old age $\mathrm{e}^{\mathrm{b})}$ & $2.145(1.019-4.816)$ & 0.042 & $0.832(0.347-2.121)$ & 0.863 \\
\hline Premorbidityc) & $3.012(1.139-8.147)$ & 0.012 & $0.792(0.891-1.241)$ & 0.376 \\
\hline Smoking & $2.682(1.102-6.781)$ & 0.028 & $1.255(0.373-9.741)$ & 0.638 \\
\hline
\end{tabular}

Table 6. Functional outcomes in laryngeal preserving cases

\begin{tabular}{|c|c|c|}
\hline Functional outcome & $\begin{array}{c}\text { Cutaneous free } \\
\text { flap }(n=44)\end{array}$ & $\begin{array}{l}\text { Pectoralis major } \\
\text { flap }(n=2)\end{array}$ \\
\hline \multicolumn{3}{|l|}{ Swallowing function } \\
\hline Any food without difficulty & $36(81.8)$ & $2(100.0)$ \\
\hline Solid food with difficulty & $2(4$ & 0 \\
\hline Semisolid or liquid food only & $5(1$ & 0 \\
\hline Impossible & $1(2.3)$ & 0 \\
\hline Decannulation & $34(77.2)$ & $2(100.0)$ \\
\hline Time of decannulation (day) & $23.5(14-150)^{b)}$ & $53.5(23-84)$ \\
\hline
\end{tabular}

$0.05)$ except postoperative atelectasis $(\mathrm{P}>0.05)$. Statistically speaking, postoperative pneumonia was more likely to occur in patients with pulmonary premorbidities, followed by smoking history, greater operative duration, and old age in that order of risk (Table 5).

The mean length of hospitalization was 39.2 days for the total cohort. It was slightly longer in the cutaneous free flap group compared to the PMMC flap group (39.4 days vs. 38.2 days), but this difference was not statistically significant.

Oral re-alimentation was achieved in 82 patients (82.8\%) within a mean of 19.2 days (range, 9 to 85 days). Gastrostomy tube dependence was total in 17 patients (17.2\%) and was slightly more likely in the cutaneous free flap group than the PMMC flap group ( 15 cases, $17.6 \%$ vs. 2 cases, $14.3 \%$; respectively, P > $0.05)$.

In laryngeal preservation cases, oral re-alimentation was accomplished in 40 patients (87.0\%) within a mean of 19.8 days (range, 9 to 85 days). Gastrostomy tube dependence was total in 6 patients (13.0\%) and only occurred in the cutaneous free flap group ( 6 cases, $13.6 \%$ vs. 0 in the PMMC flap group). The laryngeal preservation cases had slightly delayed mean oral re-alimentation starts and a low rate of gastrostomy tube dependence compared to the total cohort, but these differences did not reach 
statistical significance. Forty patients (87.0\%) showed favorable swallowing results. Decannulation was achieved in 36 patients (78.3\%) within a mean of 28.3 days (range, 14 to 150 days), whereas 10 patients $(21.7 \%)$ required a tracheotomy tube. The median decannulation time was earlier in the cutaneous free flap group compared with the PMMC flap group (23.5 days vs. 53.5 days, $P=0.254$ ), but this difference was not statistically significant. The functional outcomes of laryngeal preservation were tested using Fisher exact test and the Mann-Whitney test (Table 6).

\section{DISCUSSION}

In this retrospective comparative study of cutaneous free and PMMC flaps, we stratified patients and procedures to objectively assess functional outcomes and investigate possible correlations between preoperative risk factors and postoperative morbidities.

High incidences of overall wound complications and dehiscence at recipient sites were found in the PMMC flap group. However, it should be taken into consideration that PMMC flaps were usually selected to shorten the operative duration due to a poor general condition caused by severe preoperative comorbidities. There is widespread agreement in the literature that risk factors themselves are not associated with free flap complications [12-14]. While we did suppose that risk factor severity (e.g., degree of hypertension, degree of control of diabetes) was related to flaprelated complication rates, we did not perform a statistical analysis to assess this possibility. O'Neill et al. [15] found that the incidence of dehiscence was significantly higher in a group receiving a PMMC flap $(\mathrm{n}=2,5.4 \%)$ than in one receiving an RFFF $(n=0, P=0.040)$, but they did not speculate on a reason for this observation. You et al. [6] reported that a low preoperative albumin level $(3.8 \mathrm{~g} / \mathrm{dL})$ was not significantly correlated with high incidence rates of overall wound complications or dehiscence in the PMMC flap group of their study.

With regard to postoperative morbidity, the incidence of pneumonia was significantly higher in the cutaneous free flap group $(\mathrm{P}=0.020)$. O'Neill et al. [15] reported that the incidence of postoperative atelectasis was significantly higher in the RFFF group and hypothesized that it could be influenced by a longer operative duration. In our study, only operative duration was identified as a statistically significant risk factor for postoperative morbidity, but it only correlated with postoperative pneumonia, not atelectasis. It is well known that older age and premorbid conditions (e.g., smoking history and underlying disease) influence postoperative medical morbidity [15-17]. For example, older patients with pulmonary premorbidities would be more sensitive to the effects of prolonged anesthesia and could develop more severe complications, especially with regard to respiratory performance. Chick et al. [17] reported that chronological age alone is not a risk factor in a free flap procedure, but physiologic age after stratifying by premorbid conditions is a significant risk factor. Among the four risk factors we assessed, the strongest relationship was between pulmonary premorbidity and postoperative pneumonia. However, there was a limitation in that we did not examine individual differences.

The functional evaluation did not show meaningful differences between the cutaneous free flap group and PMMC flap group. Chepeha et al. [2] recently reported that an RFFF group had better functional outcomes than a PMMC flap group. Although many studies have reported that free flaps are superior, we found no evidence for this hypothesis in our study.

With regard to swallowing function, extensive resection of the base of the tongue or total tongue resection tended to increase the rate of tube dependency and affected oral feeding, and this should be considered in interpreting results. A limitation of our study is that an immobile tongue precluded swallowing function assessment.

Joo et al. [18] reported that $84 \%$ of 43 patients who underwent larynx-preserving partial hypopharyngectomy for hypopharyngeal carcinoma were able to meet their nutritional needs orally, and $88 \%$ were successfully decannulated. In our study, oral diets were possible in 82 patients (82.8\%) and 40 patients (87.0\%) were able to obtain all of their nutritional needs orally without restriction. Overall, 78.3\% of all subjects who underwent laryngeal preservation were able to undergo decannulation. Patients who underwent laryngeal preservation as an initial treatment had good functional outcomes.

As mentioned above, the incidence of flap-related complications was higher in the PMMC flap group, but the rate of postoperative medical morbidity was significantly lower compared to the cutaneous free flap group. It is clinically significant that the PMMC flap group had a low incidence of postoperative medical morbidities, given that these patients had poor preoperative conditions and could not tolerate long operations. The advantages of the PMMC flap with regard to lower medical morbidity should not be undervalued. Our results suggest that PMMC flaps should be considered for hypopharyngeal reconstruction, especially in patients with pulmonary issues. Moreover, cutaneous free flaps are not appropriate for patients with a poor general condition and pulmonary comorbidities.

Given the level of experience of the single reconstructive team at our medical institution, our results should be more reliable than those of studies evaluating multiple reconstructive teams' surgical outcomes by minimizing the effect of the learning curve and measurement bias, as shown in other studies. Our head and 
neck reconstruction center suggests the following guidelines for hypopharyngeal reconstruction: (1) When possible, laryngeal preservation should be prioritized in patients undergoing cancer ablation. (2) The PMMC flap is more favorable for patients with a possibly lethal premorbidity, especially pulmonary premorbidity when a long operation is impossible and a small defect is expected. (3) The cutaneous free flap is more favorable for patients without a lethal premorbidity when a long operation is realistic and a relatively large defect is expected. The ALT free flap should be considered specifically in cases of a large circumferential defect.

\section{REFERENCES}

1. Bianchi B, Ferri A, Ferrari S, et al. The free anterolateral thigh musculocutaneous flap for head and neck reconstruction: one surgeon's experience in 92 cases. Microsurgery 2012; 32:87-95.

2. Chepeha DB, Annich G, Pynnonen MA, et al. Pectoralis major myocutaneous flap vs revascularized free tissue transfer: complications, gastrostomy tube dependence, and hospitalization. Arch Otolaryngol Head Neck Surg 2004;130: 181-6.

3. Piazza C, Taglietti V, Nicolai P. Reconstructive options after total laryngectomy with subtotal or circumferential hypopharyngectomy and cervical esophagectomy. Curr Opin Otolaryngol Head Neck Surg 2012;20:77-88.

4. Welkoborsky HJ, Deichmuller C, Bauer L, et al. Reconstruction of large pharyngeal defects with microvascular free flaps and myocutaneous pedicled flaps. Curr Opin Otolaryngol Head Neck Surg 2013;21:318-27.

5. Song HG, Yun IS, Lee WJ, et al. Robot-assisted free flap in head and neck reconstruction. Arch Plast Surg 2013;40:353-8.

6. You YS, Chung CH, Chang YJ, et al. Analysis of 120 pectoralis major flaps for head and neck reconstruction. Arch Plast Surg 2012;39:522-7.
7. Corten EM, Schellekens PP, Hage JJ, et al. Clinical outcome after pedicled segmental pectoralis major island flaps for head and neck reconstruction. Ann Plast Surg 2009;63:292-6.

8. Cristalli G, Pellini R, Roselli R, et al. Pectoralis major myocutaneous flap for hypopharyngeal reconstruction: long-term results. J Craniofac Surg 2011;22:581-4.

9. McLean JN, Carlson GW, Losken A. The pectoralis major myocutaneous flap revisited: a reliable technique for head and neck reconstruction. Ann Plast Surg 2010;64:570-3.

10. Ariyan $S$. The pectoralis major myocutaneous flap: a versatile flap for reconstruction in the head and neck. Plast Reconstr Surg 1979;63:73-81.

11. Yang GF, Chen PJ, Gao YZ, et al. Forearm free skin flap transplantation: a report of 56 cases. 1981. Br J Plast Surg 1997; 50:162-5.

12. Lim YS, Kim JS, Kim NG, et al. Free flap reconstruction of head and neck defects after oncologic ablation: one surgeon's outcomes in 42 cases. Arch Plast Surg 2014;41:148-52.

13. Bozikov K, Arnez ZM. Factors predicting free flap complications in head and neck reconstruction. J Plast Reconstr Aesthet Surg 2006;59:737-42.

14. Bianchi B, Copelli C, Ferrari S, et al. Free flaps: outcomes and complications in head and neck reconstructions. J Craniomaxillofac Surg 2009;37:438-42.

15. O’Neill JP, Shine N, Eadie PA, et al. Free tissue transfer versus pedicled flap reconstruction of head and neck malignancy defects. Ir J Med Sci 2010;179:337-43.

16. Shestak KC, Jones NF. Microsurgical free-tissue transfer in the elderly patient. Plast Reconstr Surg 1991;88:259-63.

17. Chick LR, Walton RL, Reus W, et al. Free flaps in the elderly. Plast Reconstr Surg 1992;90:87-94.

18. Joo YH, Cho KJ, Park JO, et al. Role of larynx-preserving partial hypopharyngectomy with and without postoperative radiotherapy for squamous cell carcinoma of the hypopharynx. Oral Oncol 2012;48:168-72. 